\title{
Solar Flare Cosmic Rays at and beyond the Modulation Boundary
}

\author{
J. R. Joxipit and E. C. Stone \\ Downs Laboratory, California Institute of Technology \\ Pasadena, California 91109
}

In this letter we consider the characteristics of solar flare cosmic ray events observed beyond the postulated modulation boundary, should such a boundary exist. We point out that observations of solar flare particles at large distances from the sun provide a sensitive and precise diagnostic of any such boundary. The present consensus based primarily on indirect inferences from cosmic ray data appears to be that cosmic ray scattering effectively ceases within some 5 to $10 \mathrm{AU}$ [see, e.g., Burlaga, 1967; O'Gallagher and Simpson, 1967; $\mathrm{Ng}$ and Gleeson, 1971; and Garrard, 1972]. Since it is expected that the solar wind continues to flow out to perhaps 50 AU [Axford, 1971], this view implies a large region of flow in which one has the classical large-scale magnetic field [Parker, 1963], but an effective absence of irregularities, which scatters the particles. Dynamic models of the solar wind producing such phenomena have been considered by Jokipii and Davis [1969] and Jokipii [1973].

Consider then a classical solar flare event in which particle transport is due to diffusion, convection, and adiabatic deceleration. Particles with an energy spectrum $A T^{-\gamma}$ are accelerated at the sun and injected impulsively into the interplanetary medium. Within the boundary $r=D$ the particles satisfy the usual FokkerPlanck equation [Parker, 1965]

$$
\begin{array}{r}
\frac{\partial U}{\partial t}=\frac{\partial}{\partial x_{i}}\left[\kappa_{i} \frac{\partial U}{\partial x_{i}}\right]-\frac{\partial}{\partial x_{i}}\left(V_{i} U\right) \\
+\frac{1}{3}(\nabla \cdot \mathrm{V}) \frac{\partial}{\partial T}(\alpha T U)
\end{array}
$$

with the streaming flux given by [Gleeson and Axford, 1967; Jokipii and Parker, 1970]

$$
\begin{aligned}
F_{i}=-\kappa_{i i} & \partial U / \partial x_{i} \\
& +V_{i}\left[U-\frac{1}{3} \partial / \partial T(\alpha T U)\right]
\end{aligned}
$$

Copyright $\odot 1973$ by the American Geophysical Union. where $\kappa_{i j}$ is the diffusion tensor, $U$ is the density of solar cosmic rays $\left(\mathrm{cm}^{-3} \mathrm{Mev}^{-1}\right), T$ is the kinetic energy, $\alpha(T)=\left(T+2 M_{0} c^{2}\right) /\left(T+M_{0} c^{2}\right)$, and $V$ is the solar wind velocity. At the boundary $r=D$ one applies the boundary condition corresponding to free escape, $U(r=D)=0$. Small corrections to this boundary condition were calculated by Jokipii and Parker [1970], but they are not normally of any practical importance. It is, of course, not necessary that there be a sharp boundary. The possibility of a more diffuse boundary is discussed below; our conclusions are not sensitive to the assumption of a sharp boundary, which is used here for clarity.

Progressively more sophisticated and realistic solutions to these equations have been obtained by a number of authors over the last few years [Parker, 1965; Burlaga, 1967; Fisk and Axford, 1968; Forman, 1971; $\mathrm{Ng}$ and Gleeson, 1971; Lupton and Stone, 1973]. The recent solution of Lupton and Stone [1973], for example, has been quite successful in interpreting observations, and we will use it below. But we are primarily interested here in the flux of cosmic rays observed at a point beyond $r=D$. This question is of more than just academic interest, since it is quite possible that deep space probes will penetrate the boundary in the not too distant future. In fact, it is likely that measurements of the character of solar flare particle events as a function of heliocentric radius $r$ may serve as a very useful and precise diagnostic to help determine the position of the boundary or to determine its extent if the boundary is diffuse.

The characteristics of solar flare particles outside of a diffusing region were previously discussed in the context of a phenomenological one-dimensional model (neglecting energy change) for anisotropic solar cosmic ray propagation [Burlaga, 1970]. Burlaga suggested that solar cosmic rays should be anisotropic beyond 
the diffusing region, although there was no estimate of the intensity of such fluxes to determine whether they were observable.

\section{Computation of Particle Flux through THE BOJNDARY}

The characteristics of solar flare produced cosmic rays observed near earth suggest that the particles are stored in a scattering region with a heliocentric radius, $D \lesssim 10 \mathrm{AU}$. The nearly exponential decay of the isotropic flux with time is due to two effects, the leakage of the particles out of the storage region, and the slow adiabatic cooling effects occurring in the expanding solar wind. Consider now what would be the characteristics of solar cosmic rays observed at the boundary.

To illustrate the nature of the phenomenon, we will first make a very crude estimate of the general magnitude of flux of particles leaking out of the boundary, assuming that the density decay is entirely due to such leakage. The effects of adiabatic deceleration will be introduced later. For simplicity we will assume that particle density $n\left(\mathrm{~cm}^{-3}\right)$ is essentially uniform within the heliocentric sphere of radius $D$ and that the density is decaying exponentially with a time constant $\tau$. If $N$ is the total number of particles stored in the scattering region, then $d N / d t=-N / \tau$. Since in this example the density decay is due to leakage out of the boundary surface, the net flux $f_{D}\left(\mathrm{~cm}^{-2} \mathrm{sec}^{-1}\right)$ through the surface must be given by

$$
f_{D}=-\left(1 / 4 \pi D^{2}\right) d N / d t
$$

or

$$
f_{D}=D n / 3 \tau
$$

where $N=(4 / 3) \pi D^{s} n$. To compare this crude estimate of the leakage flux with the assumed flux inside $D$, recall that $n=4 \pi j / v$, where $v$ is the particle velocity and $j$ is the flux per unit solid angle. If $j(1)$ is the flux of $1-\mathrm{Mev}$ protons inside $r=D, \tau \simeq 60$ hours, and $D=10 \mathrm{AU}$, we find

$$
f_{D}=2.1 j(1)
$$

Thus, according to this very crude estimate, the flux emerging from the boundary may be comparable to that inside and may therefore be easily observable with typical currently available instruments.

As a further illustration, a more accurate estimate of the leakage flux can be made by using a full solution to equation 1 , which includes the effects of diffusion, convection, and adiabatic deceleration [Lupton and Stone, 1973]. According to this solution, the density distribution of particles and the exponential decay time depend upon the boundary distance $D$, the interplanetary diffusion coefficient $\kappa$, the solar wind velocity, and the power law index $\gamma$, which describes the kinetic energy spectrum of the particles $\left(d U / d T \sim T^{-r}\right)$. From this solution we can determine during the decay phase of an event, (a) the decay rate of the particle density at any given energy as a function of time, and (b) that portion of the decay rate due to adiabatic deceleration. Since there are no other contributors to the decay, the difference between (a) and (b) must be the rate at which particles are lost from the boundary. Hence the outward flux of particles per square centimeter can be determined. Table 1 lists the calculated decay times and the leakage fluxes for a typical range of values for these parameters. The calculated leakage flux $f_{D}$ is given in relation to $j_{m}(1)$, the calculated directional flux of $1-\mathrm{Mev}$

\begin{tabular}{|c|c|c|c|c|c|c|}
\hline \multirow{2}{*}{$\begin{array}{c}\text { Boundary } \\
\text { Distance } \\
D, \mathrm{AU}\end{array}$} & \multirow{2}{*}{$\begin{array}{c}\text { Solar Wind } \\
\text { Velocity, } \\
\text { knt/sec }\end{array}$} & \multirow{2}{*}{$\begin{array}{c}\text { Diffusion } \\
\text { Coefficient } \\
\kappa, \mathrm{cm}^{2} / \mathrm{sec}\end{array}$} & \multirow{2}{*}{$\begin{array}{c}\text { Spectral } \\
\text { Index } \\
\gamma\end{array}$} & \multirow[b]{2}{*}{$\begin{array}{c}\text { Decay } \\
\text { Time } \\
\tau, \text { hours }\end{array}$} & \multicolumn{2}{|c|}{ Leakage Flux of 1-Mev Protons } \\
\hline & & & & & $\underset{\mathrm{cm}^{-2} \sec ^{-1}}{f_{n^{2}}}$ & $\begin{array}{c}j_{D}(\min ) \\
\mathrm{cm}^{-2} \sec ^{-1} \mathbf{s r}^{-1}\end{array}$ \\
\hline $\begin{array}{l}4 \\
4 \\
4 \\
5 \\
6\end{array}$ & $\begin{array}{l}400 \\
400 \\
325 \\
300 \\
400\end{array}$ & $\begin{array}{c}2 \times 10^{20} \\
3 \times 10^{21} \\
3 \times 10^{20} \\
10^{22} \\
1.4 \times 10^{21}\end{array}$ & $\begin{array}{l}3 \\
3 \\
4.74 \\
3 \\
4.44\end{array}$ & $\begin{array}{l}41 \\
20 \\
49 \\
13 \\
40\end{array}$ & $\begin{array}{c}40 j_{E}(1) \\
0.95 j_{E}(1) \\
6.3 j_{E}(1) \\
1.6 j_{E}(1) \\
1.9 j_{E}(1)\end{array}$ & $\begin{array}{c}13 j_{E}(1) \\
0.30 j_{E}(1) \\
2.0 j_{E}(1) \\
0.51 j_{E}(1) \\
0.61 j_{E}(1)\end{array}$ \\
\hline
\end{tabular}

TABLE 1. Estimated Leakage Fluxes Calculated by Using the Full Solution to the Fokker-Planck Equation 
protons at earth. A minimum leakage flux per unit solid angle $\left(j_{D}(\min )\right)$ is calculated assuming that the flux immediately outside $D$ is isotropic over the $2 \pi$ outward directed solid angle, so that $j_{D}(\min )=f_{D} / \pi$. As is discussed below, if the flux is anisotropic outside $D$, then in the allowed directions the directional flux must be larger than $j_{D}$ (min), so that the total integrated leakage flux is equal to $f_{D}$.

As with the cruder estimate of $f_{D}$ we find that the leakage flux may well be comparable to that at earth and hence be of observable intensity. In fact, since the decay time is fixed by observation, it would seem that, even if the numerical factors in Table 1 are modified in the future by better estimates, the leakage flux should in general be observable provided that our present estimate of the relative contribution of leakage to the total observed decay rate is not in error by a large factor. Similarly, the magnitude of the leakage flux in the vicinity of a diffuse boundary should be of the same general order as that illustrated in Table 1.

We have carried out similar calculations for relativistic $(\sim 1 \mathrm{Gev})$ protons and find that $j_{D}(\min ) \sim 0.02 j_{k}$ at these energies. Hence the fluxes at these higher energies may be unobservable at the boundary. Physically, the flux at the boundary is large at low energies because, since $\boldsymbol{k}$ is small, the particle distribution during the decay phase is peaked sharply near the boundary [see, e.g., Lupton and Stone, 1973]. At higher energies, where $\kappa$ becomes larger, the particle distribution is peaked at $r \simeq 1 / 2 D$.

\section{Cosmic Ray Flux beyond the Modulation Boundary}

The cosmic ray particles beyond $r=D$ will move outward along the magnetic field lines and thus conserve their adiabatic invariants. The angular distribution will hence be highly anisotropic and directed outward along the lines of force. There will be no particles returning toward the sun. One may obtain the flux of particles as a function of distance by application of Liouville's theorem. Thus, if $j(T, \Omega$, $t) d \Omega$ is the flux of particles of energy $T$ in a given solid angle $d \Omega$ as a function of time $t$, then Liouville's theorem tells us that flux is constant along allowed trajectories. We restrict consideration to the case where the time scale of the flux variations at the boundary is much larger than the transit times of particles from the boundary to the point of observation. We can then say that, if $j_{D}(T, \Omega, t)$ is the flux at the boundary, and if $B_{0} / B_{0}$ is the ratio of magnetic field intensity at the point of observation to that at $r=D$, then the observed flux is

$$
\begin{array}{ll}
j_{0}(T, \Omega, t)=j_{D}\left(T, \Omega^{\prime}, t\right) & \theta \leq \theta_{c} \\
j_{0}(T, \Omega, t)=0 & \theta>\theta_{c}
\end{array}
$$

where $\Omega^{\prime}$ is that direction along a particle trajectory at $r=D$ that leads to $\Omega$ at the point of observation and where $\theta_{0}=\sin ^{-1}$ $\left[\left(B_{0} / B_{D}\right)^{1 / 2}\right]$ is the critical pitch angle. The zero flux for $\theta>\theta_{0}$ states simply that pitch angles greater than $\theta_{0}$ do not correspond to allowed trajectories from the boundary. For example, if $j_{D}$ were isotropic over the outward hemisphere, $j_{0}$ would be isotropic within $\theta=\theta_{\text {。 }}$ and 0 outside.

From (3) one may compute the omnidirectional flux

$$
I(T, t)=\int_{0}^{\theta_{0}} \sin \theta d \theta \int_{0}^{2 \pi} d \varphi j_{0}(T, \Omega, t)
$$

The next step is to determine $j_{D}\left(T, \Omega^{\prime}, t\right)$. The leakage flux $f_{D}$ computed in the previous section is clearly related to $j_{D}$. In fact, if $f_{D}$ is the computed outward flux at the boundary, as is defined above, $\theta_{1}$ is the angle between the particle velocity and the radius vector, and $\varphi$ is azimuth about the radius vector, we have

$$
\int_{0}^{2 \pi} d \varphi \int_{0}^{\pi} d \theta_{1} j_{D} \cos \theta_{1} \sin \theta_{1}=f_{D}
$$

Thus, if we know the dependence of $j_{p}$ on $\theta_{1}$, we can determine the flux anywhere outside the scattering region.

We first consider the case for which $j_{D}$ is isotropic over the $2 \pi$ outward directed solid angle. The directional flux in this case is the minimum directional flux corresponding to a given leakage flux $f_{D}$. We find from (5) that

$$
j_{D}(\min )=f_{D} / \pi
$$

The minimum directional fluxes corresponding to typical estimated leakage fluxes are included in Table 1.

Let us next consider a special case to illustrate the range of the phenomena. Suppose that the magnetic field beyond $r=D$ is essentially 
radial, so that $B_{0} / B_{\mathrm{D}} \simeq(D / r)^{2}$, and let $j_{D}$ be isotropic over the outward pointing hemisphere. We have $\sin \theta_{0}=D / r$ so that at a heliocentric radius $r>D, j=j_{D}$ within $\theta_{0}=\sin ^{-1}(D / r)$. The omnidirectional flux $I$ is then evaluated by using (4). One obtains

$$
\begin{aligned}
I(r, T, t) & =\frac{f_{D}(T, t)}{\pi}\left(1-\cos \theta_{c}\right) \\
& =\frac{f_{D}(T, t)}{\pi}\left[1-\left(1-\frac{D^{2}}{r^{2}}\right)^{1 / 2}\right]
\end{aligned}
$$

Thus, at large $r, I$ falls off as $1 / r^{2}$. It is clear that this calculation also applies to the case where the region beyond $r=D$ is field free, so that particles move in straight lines.

It is the radial flux that is fixed by the decay time $\tau$. If, as is expected, the field beyond the boundary is inclined at the Parker spiral angle $\psi$ relative to the radial direction, the directional flux should be even larger, since the radial velocity of the particles is decreased by a factor of the order of $\cos \psi$. To maintain the same radial flux, the density should therefore be increased by a factor of the order of $(\cos \psi)^{-1}$. So we expect that, for a given decay time $\tau$, the directional fluxes may be even larger than those calculated here. In addition, because of the spiral field the radial decrease of the flux will be slower than that given in (6).

\section{EFFect of a Diffuse Boundary}

Although it is not our intent to quantitatively describe the effects of a diffuse boundary region on the intensity and anisotropy of the decay phase of flare events, several general comments seem appropriate. The flux beyond the boundary region in an essentially scatter free medium should be $\sim 100 \%$ anisotropic, independent of the width of the boundary region. The flux sunward of the boundary region should be essentially isotropic during the decay phase, also independent of the width of the boundary region. In the transition region then, the flux must become increasingly anisotropic with the transition from the inner scattering region to the outer scatter free region. Thus detailed anisotropy measurements within the boundary transition region should provide valuable information on the spatial dependence of the decrease of the magnetic irregularities that are causing the scattering.

\section{Discussion and Conclusions}

In this letter we have presented illustrative calculations of the flux of solar cosmic rays observed beyond the boundary of the modulation region, if it is assumed that such a boundary exists. Such considerations are of considerable interest in view of the probable penetration of deep space probes to distances of the order of $10 \mathrm{AU}$ in the near future.

Reasonable estimates of fluxes indicate that classical solar cosmic ray events should be readily observable well beyond the boundary with typical existing instrumentation. Since solar cosmic rays observed beyond the modulation boundary should be $\sim 100 \%$ anisotropic throughout the decay phase, whereas those inside the boundary should be essentially isotropic, observations of such events should provide a valuable diagnostic to help determine the location and extent of such a boundary. The occurrence of consistently high anisotropies during the decay phase of solar cosmic ray events provides a 'signature' of the boundary. Thus the observation of classic nearly isotropic decay phases out to any given heliocentric distance would be strong evidence that any free escape boundary must be further out. Conversely, observation of $\sim 100 \%$ anisotropic decays implies that the point of observation is beyond the point at which particles are scattered.

Acknowledgments. We acknowledge the assistance of F. Marshall in calculating the numbers in Table 1. One of us (E.C.S.) is an Alfred P. Sloan Foundation Fellow.

This work has been supported in part by the National Aeronautics and Space Administration under grant NGR 05-002-160 and by the Alfred P. Sloan Foundation.

\section{REFERENCES}

Axford, W. I., Interaction of the Solar Wind with the Interstellar Medium, paper presented at Solar Wind Conference, Univ. of Calif. and NASA Ames Res. Center, Pacific Grove, Calif., March 21-26, 1971.

Burlaga, L. F., Anisotropic diffusion of solar cosmic rays, J. Geophys. Res., 72, 4449, 1967.

Burlaga, L. F., Anisotropic solar cosmic ray propagation in an inhomogeneous medium, part 2, Solar Phys., 12, 317, 1970.

Fisk, L., and W. I. Axford, Effect of energy change on solar cosmic rays, J. Geophys. Res., 73, 4396, 1968.

Forman, M. A., Convection-dominated transport of solar cosmic rays, J. Geophys. Res., 76, 759, 1971. 
Garrard, T. L., A quantitative investigation of the solar modulation of cosmic-ray protons and helium nuclei, Ph.D. thesis, Calif. Inst. of Technol., Pasadena, 1972.

Gleeson, L. J., and W. I. Axford, Cosmic rays in the interplanetary medium, Astrophys. J., 149, L1115, 1967.

Jokipii, J. R., Radial variation of magnetic fluctuations and the cosmic-ray diffusion tensor in the solar wind, Astrophys. $J$, in press, 1973.

Jokipii, J. R., and L. Davis, Jr., Long-wavelength turbulence and the heating of the solar wind, Astrophys. J., 156, 1101, 1969.

Jokipii, J. R., and E. N. Parker, On the convection, diffusion, and adiabatic deceleration of cosmic rays in the solar wind, Astrophys. $J$., 160, 735, 1970.

Lupton, J., and E. C. Stone, Solar flare particle propagation: Comparison of a new analytic solution with spacecraft measurements, $J$. Geophys. Res., 78, 1007, 1973.

Ng, C. K., and L. J. Gleeson, On the propagation of solar cosmic rays, Proc. Int. Conf. Cosmic Rays, 14th, \&, 498, 1971.

O'Gallagher, J. J., and J. A. Simpson, The heliocentric intensity gradients of cosmic-ray protons and helium during minimum solar modulation, Astrophys. J., 147, 819, 1967.

Parker, E. N., Interplanetary Dynamical Processes, pp. 137-140, Interscience, New York, 1963.

Parker, E. N., The passage of energetic charged particles through interplanetary space, Planet. Space Sci., 13, 9, 1965.

(Received December 7, 1972; accepted January 26, 1973.) 